\title{
Primary intracranial multiple hydatid cysts in an adult
}

Vandana Sharma, Anuja Sharma, Mahima Sharma, Anil Sharma1, Arvind Khajuria

Department of Pathology, Acharya Shri Chander College

of Medical Sciences and Hospital, Sidhra, ${ }^{1}$ Department of Neurosurgery, Jammu Brain Spine

Trauma Centre, Jammu, Jammu and Kashmir, India

Address for the Correspondence:

Dr. Mahima Sharma, House No.-109, Sector-5, Trikuta Nagar, Jammu - 180 012,Jammu and Kashmir, India.

E-mail: anujshrm@rediff.com

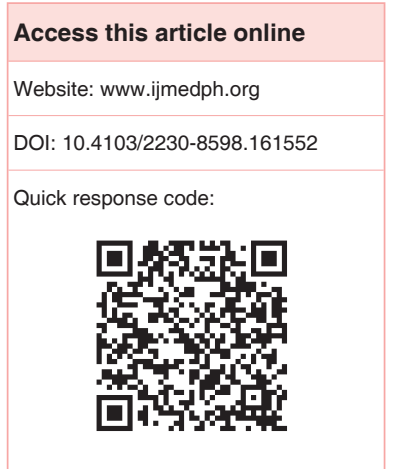

Cerebral hydatid disease is an uncommon presentation of echinococcosis representing 1-2\% of all Echinococcus granulosus infestations. Most of the cases are seen in children. Primary multiple hydatid disease of the brain in adults is a rare entity. We report here a case of 35-year-old male with primary cerebral hydatid disease without extracranial lesions.

Key words: Echinococcosis, intracranial, primary hydatid cyst

\section{INTRODUCTION}

Hydatid disease (echinococcosis) is a worldwide zoonosis produced by the larval stage of the Echinococcus tapeworm. ${ }^{[1]}$ The definite host of Echinococcus are various carnivores, the dog being the most common and the intermediate hosts are sheep, goats, and cattle. ${ }^{[2,3]}$ Humans get infected through feco-oral route, by the ingestion of food or milk contaminated by dog feces containing ova of the parasite or by direct contact with dogs. ${ }^{[2,3]}$ The disease is endemic in many parts of the world particularly in Middle East, Australia, New Zealand, South America, Central and South Europe as well as in Turkey. ${ }^{[1,3]}$ In India, the hydatid disease is more commonly seen in parts of Andhra Pradesh, Tamil Nadu and Punjab. ${ }^{[3]}$ Hydatid disease most commonly involves liver (50-75\%) and lungs $(10-40 \%) .^{[1,4]}$ The remaining lesions may involve any organ of the body including brain with only $2 \%$ of the patients infected. ${ }^{[1]}$ Cerebral hydatidosis is primarily associated with involvement of liver or lungs but an isolated infestation of the central nervous system has also been reported. ${ }^{[5]}$ Primary cerebral hydatid disease is a rare entity. ${ }^{[1-3]}$

\section{CASE REPORT}

A 35-year-old man presented with features of raised intracranial pressure and rapidly developing left hemiparesis. A non-contrast computed tomography (CT) scan of the head showed presence of two hypodense lesions, one in right occipital region and another one situated deeply causing compression of the occipital horn and body of lateral ventricle. Magnetic resonance imaging (MRI) of brain with contrast showed the presence of the same lesions and another small lesion in the parietal region of the left cerebral hemisphere [Figure 1]. A diagnosis of multiple hydatid cysts of the brain involving both cerebral hemispheres was suggested. Extensive work-up of the patient failed to reveal any evidence of primary disease in other organs. The patient was planned for elective surgery but patient developed rapid deterioration of higher mental functions. He was admitted and put on steroids, anticonvulsants and was taken for urgent craniotomy after $24 \mathrm{~h}$. Right occipital free flap craniotomy was done and dura opened with flaps based on superior sagittal sinus and transverse sinus. The method of Dowling technique was adopted to remove the cyst. Because of the shear size of cyst and raised intracranial pressure, the cyst wall ruptured during the surgery, although there was no spillage. The resultant cavity was washed for any residual membrane. No attempt was made to remove the deeper cyst of the right cerebral hemisphere considering the patient's status peroperatively. Postoperative recovery was uneventful. The patient was reoperated after 1-month to remove the deeper cyst. Patient had rapid neurological recovery. To prevent recurrence and to treat the smaller cyst in left cerebral hemisphere, patient was put on long term albendazole and anti-convulsants. Postoperative CT scan following both the surgeries showed encephalomalacic changes with no mass effect [Figure 2]. 


\section{Patholgical findings}

Grossly, glistening white, thin-walled cyst was seen. Histopathological examination of the cyst revealed typical laminated cyst wall lined by germinal layer with brood capsules and scolices [Figure 3]; confirming the CT diagnosis of hydatid cyst.

\section{DISCUSSION}

Hydatid disease is caused by the larval stage of Echinococcus granulosus. ${ }^{[1-3]}$ Hydatidosis is usually seen in endemic areas of cattleraising countries. ${ }^{[1,3,6]}$ Though, hydatid cyst can occur in any organ or tissue of the body, the bone and brain are the fourth common site of involvement after liver, lungs, and peritoneum. ${ }^{[2]}$ Intracranial hydatid cysts account for 1-2\% of all Echinococcus infestations with or without associated cyst in other organs of the body. ${ }^{[1-3,5,7,8]}$

Cerebral echinococcosis is most commonly seen in children and young adults. ${ }^{[1-9]}$ Intracranial hydatid cysts are usually seen in the supratentorial compartment; in the territory supplied by the middle cerebral artery. ${ }^{[2-8]}$ The parietal lobe is the most frequently involved region..$^{[2-8]}$ Less common sites are the skull, cavernous sinus, eyeball, pons, cerebellum and ventricles. ${ }^{[3,4]}$

Intracranial hydatid cysts may be classified as primary and secondary. ${ }^{[3,7]}$ The primary hydatid cysts are formed as a result of direct infestation of larvae in the brain without demonstrable involvement of other organs. ${ }^{[2,3]}$ Primary cysts are fertile as they contain scolices and brood capsules, hence their rupture can result in recurrence. ${ }^{[3]}$ Secondary cysts result from spontaneous traumatic or surgical rupture of primary intracranial hydatid cyst and they lack brood capsule and scolices. ${ }^{[2,3,7]}$ Intracranial hydatid cysts are commonly solitary. ${ }^{[3,4]}$ Multiple intracranial cysts are rare. ${ }^{[2]}$ Primary hydatid cysts are usually solitary and secondary cysts are usually multiple. ${ }^{[5]}$ Primary multiple hydatid disease of the brain is a rare pathological entity and only few case reports of primary multiple hydatid cysts have been reported in world literature. ${ }^{[2,3,9]}$

The cerebral hydatid cysts are slow growing and present late when they increase in size and become large..$^{[3]}$ In adults, cardinal symptoms of focal neurological deficit in the form of hemiparesis, speech disorders, hemianopia, papilledema and epileptic seizures are seen; whereas the children show clinical symptoms of raised intracranial tension, which may be due to the large size of cyst or interference with the pathway of cerebrospinal fluid. ${ }^{[2,3,5,7,9]}$ Calcification and surrounding edema are rarely seen in cerebral hydatid cysts. ${ }^{[6]}$

The correct preoperative diagnosis can be established by CT and MRI. CT is superior in detecting calcification in the cyst, whereas MRI is better in demonstrating cyst capsule thus helps in detecting the multiplicity and also defines the anatomic relationship of the lesion with the adjacent structures. Moreover, MRI is more helpful in planning the surgery of the patient. ${ }^{[1]}$ However, specific diagnosis is made by histopatholgical examination. ${ }^{[6]}$

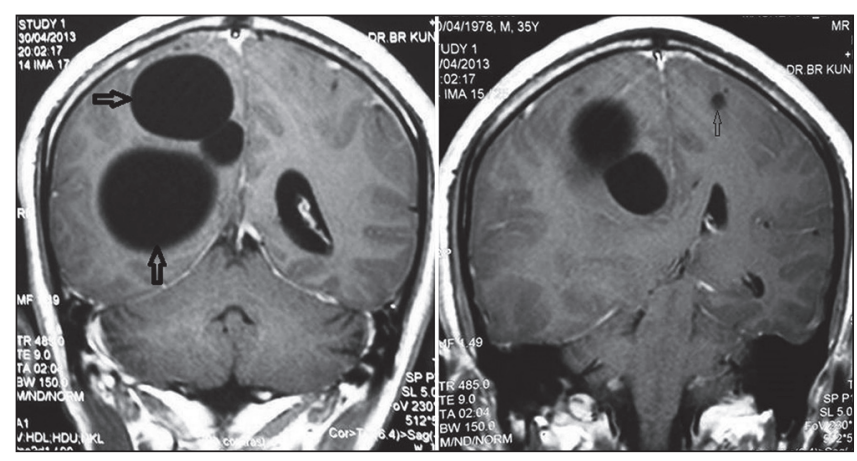

Figure 1: Magnetic resonance imaging (MRI) brain shows presence of two well defined nonenhancing cystic lesions in right parietal and occipital lobes (thick arrows) along with presence of thin septation in one of the lesion. A small cystic lesion (thin arrow) with similar MRI morphology is seen in left cerebral hemisphere. Associated mass effect is seen on lateral ventricle with contralateral midline shift and brainstem rotation

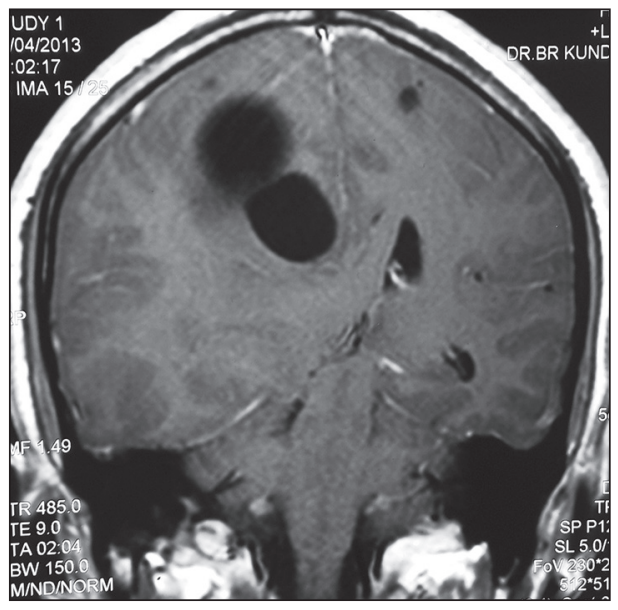

Figure 2: Computed tomography head shows complete excision of the lesion with encephalomalacic changes in parieto-occipital region with overlying bone flap. No mass effect is seen

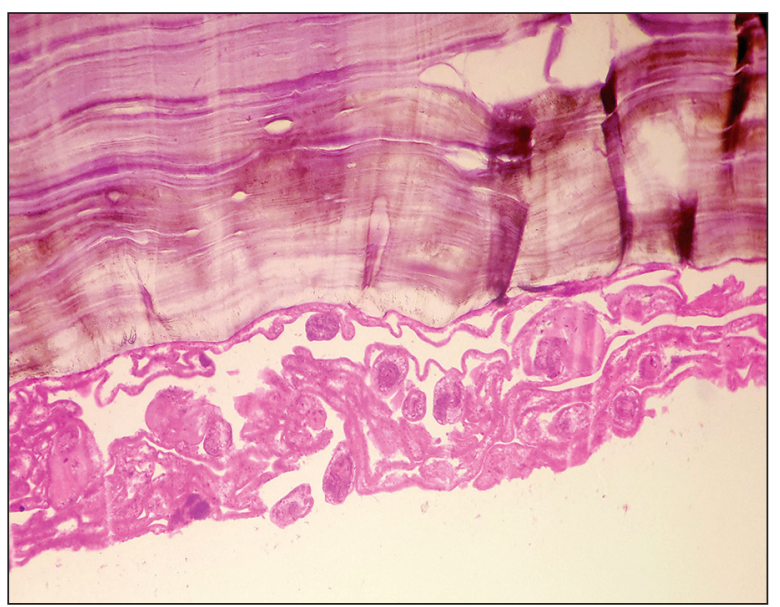

Figure 3: Section from the cyst wall shows typical laminated cyst wall lined by germinal layer with brood capsules and scolices (hematoxylin and eosin, $\times 100$ )

The differential diagnosis of cerebral hydatid cyst includes cystic lesions like porencephalic cysts, arachnoid cysts cystic tumor of the brain and pyogenic abcess. ${ }^{[1,6,9]}$ 
The treatment of hydatid cyst is exclusively surgical. ${ }^{[3,6,7]}$ The surgical challenge is to remove the cyst intact to prevent the rupture, recurrence and anaphylaxis. ${ }^{[3,6,7]}$ The preferred technique is Dowling technique and it involves valsalva maneuver, gravity assisted delivery of the cyst from the corticectomy site, use of saline irrigation to break adhesions between brain and cyst wall and utmost precautions to ensure that cyst wall does not rupture. ${ }^{[5,10]}$ A supplementary drug therapy in the form of albendazole is given for the small, multiple cysts. ${ }^{[5]}$

\section{CONCLUSION}

Cerebral hydatid cyst should be considered as an important entity in the differential diagnosis of cystic, intracranial space occupying lesions and it can safely be managed surgically. The patients need to be observed for recurrence in neurospinal axis on long term basis.

\section{REFERENCES}

1. Bükte $Y$, Kemaloglu S, Nazaroglu H, Ozkan U, Ceviz A, Simsek M. Cerebral hydatid disease: CT and MR imaging findings. Swiss Med Wkly 2004;134:459-67.
2. Ali M, Mahmood K, Khan P. Hydatid cysts of the brain. J Ayub Med Coll Abbottabad 2009;21:152-4.

3. Gupta S, Desai K, Goel A. Intracranial hydatid cyst: A report of five cases and review of literature. Neurol India 1999;47:214-7.

4. Desai P, Bhat M. Multiple cerebral hydatid cysts. Appl Radiol 2009;36:36-7

5. Tatli M, Guzel A, Altinors N. Large primary cerebral hydatid cysts in children. Neurosciences (Riyadh) 2006;11:318-21.

6. Radmenesh F, Nejat F. Primary cerebral hydatid cyst: Two cases report. Iran J Pediatr 2008;18:83-6.

7. Tyagi DK, Balasubramaniam S, Sawant HV. Primary calcified hydatid cyst of the brain. J Neurosci Rural Pract 2010;1:115-7.

8. Patankar T, Chotai N, Prasad S, Chowdhry S, Goel A. Intracerebral hydatid cyst in a child with atrial septal defect. Indian Pediatr 1999;36:406-8.

9. Razzaq AA, Hashim AS. Multiple cerebral hydatid cysts: A surgical challenge. J Pak Med Assoc 2000;50:35-7.

10. Guvenc G, Ozdemir N, Yildirim L. Successful treatment of hydatid cyst into the lateral ventricle with dowling's technique in an adult patient. J Neurol Sci Turk 2011;28:265-9.

How to cite this article: Sharma V, Sharma A, Sharma M, Sharma A, Khajuria A. Primary intracranial multiple hydatid cysts in an adult. Int J Med Public Health 2015;5:247-9.

Source of Support: Nil, Conflicts of Interest: None declared. 